\title{
Prediction of Axial Variation of Plasma Potential in Helicon Plasma Source Using Linear Regression Techniques
}

\author{
Vipin Shukla \\ Department of Electrical Engineering, \\ Pandit Deendayal Petroleum University, Gandhinagar, India. \\ Corresponding author: vipin.shukla@pdpu.ac.in \\ Mainak Bandyopadhyay \\ ITER-INDIA, \\ Institute for Plasma Research, HBNI, Gandhinagar, Gujarat, India. \\ E-mail: mainak.bandyopadhyay@iter-india.org \\ Vivek Pandya \\ Department of Electrical Engineering, \\ Pandit Deendayal Petroleum University, Gandhinagar, India. \\ E-mail: vivek.pandya@ sot.pdpu.ac.in \\ Arun Pandey \\ Negative Ion Based Neutral Beam Injection Group, \\ Institute for Plasma Research, HBNI, Gandhinagar, Gujarat, India. \\ E-mail: arun.pandey@ipr.res.in
}

(Received March 17, 2020; Accepted June 10, 2020)

\begin{abstract}
Analytical expressions are used frequently for the determination and analysis of plasma parameters. Instead of relying on analytical expressions, the proposed method uses regression techniques supplemented with experimental data for the selected parameters (plasma potential). In the machine learning domain, this is equivalent to the creation of the training data set, building and training the model, and authenticating the result over a range of desired physical parameters. An experimental dataset is built using two axially movable Triple Langmuir Probe (TLPs) which measure the electron temperature, electron density, and electric potential of a plasma. The presented work is a first step towards developing an inclusive model with detailed kinetic simulations capable of characterizing the HELicon Experiment for Negative ion source (HELEN-I) with a single driver. Plasma potential is measured at different axial locations $(z)$ by keeping pressure fixed at 6 mTorr.
\end{abstract}

Keywords- Plasma potential, Regression, Axial position, Ion source, HELEN-I.

\section{Introduction}

The ion source is an essential part of the equipment used for fusion applications. Ion sources use gases such as hydrogen, argon for producing high-density ions (Bhattacharyya, 2009). Langmuir probes are routinely used in many plasma devices for research and industrial applications, to provide measurements of electron temperature, density and electric fields, and saturation current, among other parameters (Godyak and Alexandrovich, 2015). Plasma parameters such as electron temperature, electron density and plasma potential play an essential role in the characterization of plasma. The Langmuir probe is generally used for the direct measurement of the parameters described above (Hopkins, 1995). Langmuir probes are considered the most suitable tool for the determination of these parameters due to simplicity in measuring local values. It is used in RF 
International Journal of Mathematical, Engineering and Management Sciences

Vol. 5, No. 6, 1284-1299, 2020

https://doi.org/10.33889/IJMEMS.2020.5.6.095

plasma sources that are subject to RF pickup, which can significantly distort the I-V characteristic and give erroneous results (Merlino, 2007). Unfortunately, the analytical method cannot approximate the interaction between physical parameters such as magnetic field, plasma drift, or the geometry of the ion source.

Therefore, in the light of recent advances in the area of machine learning (ML), multivariate regression techniques can play an essential role for accurate and fast estimation of plasma source parameters and can efficiently model Langmuir probe behavior for unique experimental setups and laboratory environments. Gidon et al. (2019) presented a machine learning-based technique for Real-time diagnostics of cold atmospheric plasma (CAP) sources which successfully determined the rotational and vibrational temperatures from Optical Emission Spectroscopy using linear regression, k-means clustering and Gaussian basis regression techniques. Mesbah and Graves (2019), presented supervised and unsupervised learning for transforming modelling and simulation, real-time monitoring, and control of Non-Equilibrium Plasma (NEP). Witman et al. (2019) demonstrated the deep reinforced learning method for the regulation of thermal properties of atmospheric pressure plasma jets on substrates having different thermal and electrical characteristics. Gidon et al. (2019) shows the application of convolutional neural networks (CNNs) to reconstruct the 2-D plasma profile based on the data acquired through diagnostics and also discuss the use of recurrent neural networks for predicting the plasma disruptions. Kates-Harbeck et al. (2019) presented a method based on deep learning for forecasting disruptions using the reinforced neural network which uses gradient backpropagation for learning. Kim et al. (2004) filed an empirical technique for constructing predictive models of plasma etch processes using a combination of generalised regression neural network (GRNN) and a random generator (RG). Ho et al. (2019) outlined a Gaussian process regression (GPR) for the validation of plasma turbulent transport model. Rea et al. (2018) investigates the prediction of disruption for two different plasma devices using random forest regression technique.

The literature survey for this study suggested that several researchers successfully utilized machine learning techniques (mainly artificial neural network techniques) for the prediction of different plasma properties and characteristics. However, none of the researchers has employed machine learning tools for predicting the axial variation of plasma potential.

So, the presented work considers HELicon Experiment for Negative ion source (HELEN-I) with a single driver which is a helicon plasma source developed at Institute for Plasma Research (IPR) for producing very high-density negative hydrogen plasma. The HELEN-I is meant for carrying out studies to establish large-sized, multi-driver negative helicon plasma source. The conceptual design of the device and operational parameters have been discussed in the previously reported work. In the following, we assess the feasibility of using linear regression to predict the axial variation of plasma potential. The proposed methodology uses experimental data measured using TLP to construct a linear regression model using machine learning techniques. Polynomial Linear Regression (PLR) and regression using Gaussian Basis Function (GBF) are used for the estimation of axial variation of plasma potential. The reasons behind the selection of PLR and GBF-based regression technique are substantiated in section 3.0. Results obtained with these two approaches are then presented. We conclude with a summary of our findings and concluding remarks.

\section{Experimental Setup and Training Data Sets}

The functional description of HELEN-I experimental setup is demonstrated in Pandey et al. (2017). HELEN-I is used as a test setup to validate the proposed machine learning-based technique for 
International Journal of Mathematical, Engineering and Management Sciences

Vol. 5, No. 6, 1284-1299, 2020

https://doi.org/10.33889/IJMEMS.2020.5.6.095

plasma parameter estimation since the manual characterization of HELEN-I plasma source with hydrogen gas has already been completed (Pandey et al., 2019). The HELEN-I is operated for different input settings to generate negative hydrogen plasma. The RF power is adjusted from between $400 \mathrm{Watt}$ to $950 \mathrm{Watt}$ by keeping pressure settings at $6 \mathrm{mTorr}, 7.5 \mathrm{mTorr}$ and $30 \mathrm{mTorr}$, respectively.

Further, the plasma density is enhanced by varying the magnetic fields (B field: 40 Gauss, 55 Gauss and 86 Gauss). The plasma density is measured at different locations using Two axially movable Triple Langmuir probes (TLPs) through the plasma potential in the plasma chamber. The two probes are inserted through the L-shaped bottom flange, and the other probes are inserted from the top of the source for axial measurements. The probes, made from a tungsten wire, are used for axial and radial measurements in the expansion chamber and have three identical tips. The axial profile is obtained through the rotation of the probe. Training data set is built using the measurement taken by TLP. TLP is used to measure the plasma potential at several axial locations ranging from $\mathrm{z}=0 \mathrm{~cm}$ to $30 \mathrm{~cm}$.

The $70 \%$ of the acquired experimental data is utilised for training the regression models using different ML algorithms, and the remaining data (30\%) is used for testing purpose. The axial location and plasma potentials are regarded as two independent experimentally measured datasets while applying the regression techniques. Though as a matter of fact, the axial variation of potential is correlated with other input parameters such as magnetic field, pressure, geometry, RF power. However, in this work, only the plasma potential and the axial locations are taken into account. Therefore axial position is treated as an independent variable.

\section{Methodology}

Regression analysis is generally used for establishing the relationship between a set of independent variables and a dependent variable. Numerous types of regression models can be utilised for fitting the experimental data. This selection of regression technique often depends on the nature of data available for the dependent variable. Based on which regression model is selected, which gives the best fit (Schneider et al., 2010).

Plasma potential variation is recorded at different axial locations (z) using TLP. Total 42 such measurements for axial change of plasma potential are recorded. Figure 1 shows the axial variation of plasma potential above $700 \mathrm{~W}$ RF power and $6 \mathrm{mTorr}$ pressure. Figure 1 clearly shows the nonaxial linearity in the variation.

There are several regression techniques available for estimation and prediction (Palmer and Connell, 2009). The number of independent variables, type of dependent variables and shape of the regression line is three motivating factors behind the selection of regression techniques. In the view of nonlinearity present in the plasma potential, linear regression techniques which can efficiently deal with non-linearity become the obvious choice for implementation. Lichtenberg and Simsek (2017) states that "There was no simple model that predicted well in all data sets, but in nearly all data sets, there was at least one simple model that predicted well". Hence, linear regression methods such as simple linear regression, polynomial regression and linear regression with nonlinear basis combination of basis function, are considered for the present work.

A comparative study of the linear regression methods mentioned above is given in Table 1. 
International Journal of Mathematical, Engineering and Management Sciences

Vol. 5, No. 6, 1284-1299, 2020

https://doi.org/10.33889/IJMEMS.2020.5.6.095

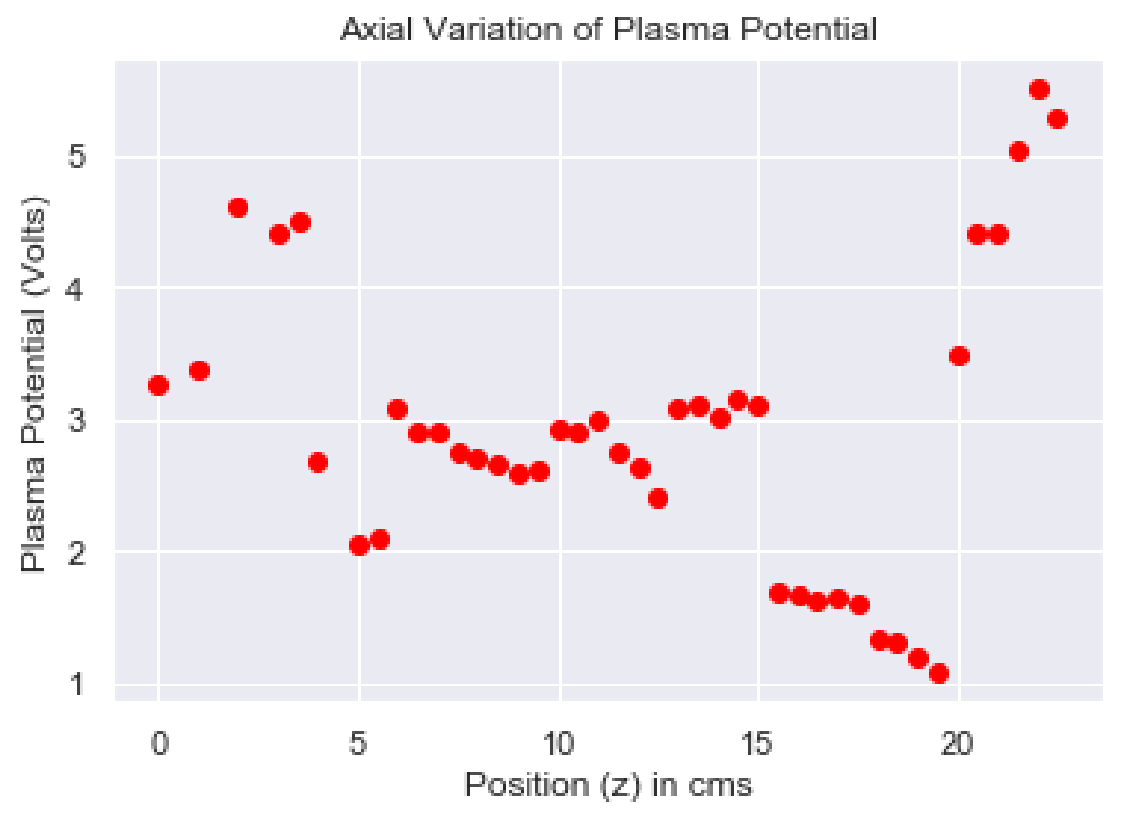

Figure 1. The scattered plasma potential (Volts) values at $\mathrm{z}=0$ to $22 \mathrm{~cm}$ locations

Table 1. Comparison of different linear regression techniques

\begin{tabular}{|c|c|c|c|}
\hline $\begin{array}{l}\text { Regression } \\
\text { Technique }\end{array}$ & Criteria of Selection & Advantages & Disadvantages \\
\hline $\begin{array}{l}\text { Simple Linear } \\
\text { Regression } \\
\text { (Schneider et al., } \\
\text { 2010) }\end{array}$ & 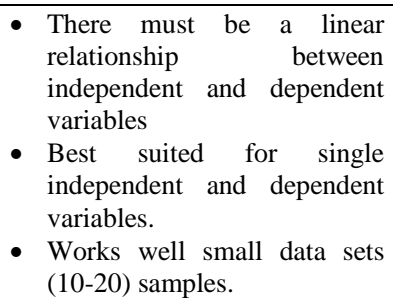 & $\begin{array}{l}\text { - } \text { Easy to implement } \\
\text { - Less number of parameters to tune } \\
\text { - No hyper-parameters to tune. }\end{array}$ & $\begin{array}{l}\text { - Linear Regression is very } \\
\text { sensitive to Outliers } \\
\text { - Only suitable for linear } \\
\text { functions }\end{array}$ \\
\hline $\begin{array}{l}\text { Polynomial } \\
\text { Linear } \\
\text { Regression } \\
\text { (Ostertagová, } \\
\text { 2012) }\end{array}$ & 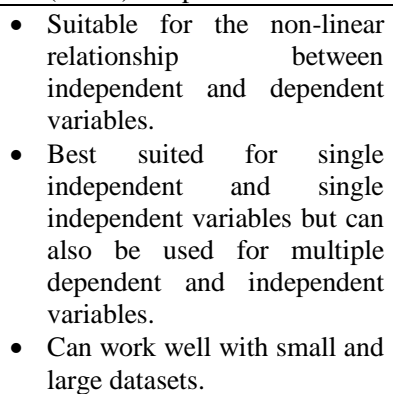 & $\begin{array}{l}\text { - Gives the improvised } \\
\text { approximation of the relationship } \\
\text { between the dependent and } \\
\text { independent variable with respect } \\
\text { to simple linear regression. } \\
\text { - Fits a wide range of curvature. } \\
\text { - Suitable for broad ranges of } \\
\text { functions. } \\
\text { - No hyperparameters to tune }\end{array}$ & $\begin{array}{l}\text { - Suffer overfitting for higher- } \\
\text { order polynomial. } \\
\text { - The presence of one or two } \\
\text { outliers in the data can } \\
\text { seriously affect the results of } \\
\text { the nonlinear analysis. } \\
\text { - fewer model validation tools } \\
\text { for the detection of outliers }\end{array}$ \\
\hline $\begin{array}{l}\text { Gaussian Basis } \\
\text { Function } \\
\text { Regression } \\
\text { (Bishop, 2006) }\end{array}$ & $\begin{array}{l}\text { - } \begin{array}{l}\text { Suitable for the non-linear } \\
\text { relationship } \\
\text { between }\end{array} \\
\text { independent and dependent } \\
\text { variables } \\
\text { - Can work well with the small } \\
\text { and large data set. } \\
\text { - Best suited for single or } \\
\text { multiple independent and } \\
\text { dependent variables. }\end{array}$ & $\begin{array}{l}\text { - Gives the best approximation of the } \\
\text { relationship between the dependent } \\
\text { and independent variable. } \\
\text { - With a combination of higher } \\
\text { dimensional Gaussian basis } \\
\text { function can fit any curvature or } \\
\text { function } \\
\text { - Regularization overcomes the } \\
\text { overfitting and cross-validation are } \\
\text { not required. }\end{array}$ & $\begin{array}{l}\text { - More hyperparameters to } \\
\text { tune. } \\
\text { - High computational cost. }\end{array}$ \\
\hline
\end{tabular}


International Journal of Mathematical, Engineering and Management Sciences

Vol. 5, No. 6, 1284-1299, 2020

https://doi.org/10.33889/IJMEMS.2020.5.6.095

The attributes tabulated in Table 1 and the nature of the experimental data set shown in Figure 1 motivates the researchers to adopt polynomial linear regression and Gaussian basis function-based regression for modelling. Applying regression by the linear combination of basis functions may prove a promising candidate for modelling (Kondor, 2004).

In this work, polynomial regression and gaussian regression techniques used in machine learning field are implemented and tested for the prediction of axial variation of plasma potential (Bishop, 2006).

\subsection{Polynomial Linear Regression}

Polynomial Regression is very similar to multiple regression but at the same time instead of having different variables higher-order power of the same variable is used as shown in equation 1. Simple linear regression given in (1) can be used for the fitting the experimental data set, i.e. axial positions $(z)$, plasma potential $\left(V_{p}\right)$ and $\varepsilon$ is the error term.

$V_{p}=\beta_{0} z^{0}+\beta_{1} z^{1}+\beta_{2} z^{2}+\ldots \ldots \ldots \ldots . . . \beta_{k} z^{k}+\varepsilon$

When the regression model given by (1) is written as $V_{p}=\beta Z+\varepsilon$ where $z$ is the coefficient matrix given by $\left(\begin{array}{ccccc}1 & z_{11}{ }^{1} & z_{12}{ }^{2} & \cdots & z_{1 k}{ }^{k} \\ 1 & z_{21}{ }^{1} & z_{22}{ }^{2} & \cdots & z_{2 k}{ }^{k} \\ \vdots & \vdots & \vdots & \ddots & \vdots \\ 1 & z_{i 1}{ }^{1} & z_{i 2}{ }^{2} & \cdots & z_{i k}{ }^{k}\end{array}\right)$ and $\hat{\beta}=\left(Z^{\prime} Z\right)^{-1} Z^{-1} V_{P}$. Considering the nature of data set given in Figure 1, finding out the order of best fit polynomial may need several trials and increase in the order of polynomial may change the value of linear, quadratic or cubic parameters. So, it is preferred to have a situation where an increase in the order of polynomial should not change the values of parameters and can be achieved by using a system of polynomials given by (2)

$V_{P_{i}}=\alpha_{0} P_{0}\left(z_{i}\right)+\alpha_{1} P_{1}\left(z_{i}\right)+\alpha_{2} P_{2}\left(z_{i}\right)+\ldots \ldots .+\alpha_{k} P_{k}\left(z_{i}\right)+\varepsilon_{i}, i=1,2,3 \ldots \ldots . n$

Though, there are several ways to construct a system of polynomials given by Gram-Schmidt method (Ramesha and Raja, 2011) that is used to create a discrete orthogonal polynomial system given in (2) Where $P_{u}\left(z_{i}\right)$ is the $u^{\text {th }}$ order polynomial defined as

$\sum_{i=1}^{n} P_{r}\left(z_{i}\right) P_{s}\left(z_{i}\right)=0, r \neq s ; r, s=0,1,2 \ldots \ldots . . k$

$P_{0}\left(z_{i}\right)=1$. In the context of $Z$ - Matrix, in this case, can be given by (4) 
International Journal of Mathematical, Engineering and Management Sciences

Vol. 5, No. 6, 1284-1299, 2020

https://doi.org/10.33889/IJMEMS.2020.5.6.095

$$
Z=\left(\begin{array}{cccc}
P_{0}\left(z_{1}\right) & P_{1}\left(z_{1}\right) & \cdots & P_{k}\left(z_{1}\right) \\
P_{0}\left(z_{2}\right) & P_{1}\left(z_{2}\right) & \cdots & P_{k}\left(z_{2}\right) \\
\vdots & \vdots & \ddots & \vdots \\
P_{0}\left(z_{n}\right) & P_{1}\left(z_{n}\right) & \cdots & P_{k}\left(z_{n}\right)
\end{array}\right)
$$

Since $X$-matrix has orthogonal columns, so $Z Z Z$ becomes

$$
Z^{\prime} Z=\left(\begin{array}{cccc}
\sum_{i=1}^{n} P_{0}^{2}\left(z_{i}\right) & 0 & \cdots & 0 \\
0 & \sum_{i=1}^{n} P_{1}^{2}\left(z_{i}\right) & \cdots & 0 \\
\vdots & \vdots & \ddots & \vdots \\
0 & 0 & \cdots & \sum_{i=1}^{n} P_{k}^{2}\left(z_{i}\right)
\end{array}\right)
$$

The ordinary least square estimator is $\hat{\alpha}=\left(Z^{\prime} Z\right)^{-1} Z^{\prime} V_{p}$ so $\hat{\alpha}_{j}(j=1,2,3 \ldots . . n)$ is given by

$$
\hat{\alpha}_{j}=\frac{\sum_{i=1}^{n} P_{j}\left(z_{i}\right) V_{P_{i}}}{\sum_{i=1}^{n} P_{j}^{2}\left(z_{i}\right)}, j=0,1,2, \ldots . . k
$$

Since $P_{0}\left(z_{i}\right)$ is a polynomial of order zero and has unit value so $\hat{\alpha}_{0}=\bar{V}_{p}$ where $\bar{V}_{P}$ is the mean value of plasma potential. The residual sum of the square is $S S_{\mathrm{Res}}(k)=\sum_{i=0}^{k}\left(V_{P_{i}}-\hat{V}_{P_{i}}\right)^{2}$ where $V_{P_{i}}$ is the observed value of plasma potential and $\hat{V}_{P_{i}}$ is the estimated value of plasma potential. The regression sum of square computed as $\frac{\left[\sum_{i=1}^{n} P_{j}\left(z_{i}\right) V_{P_{i}}\right]^{2}}{\sum_{i=1}^{n} P_{j}^{2}\left(x_{j}\right)}$ does not depend on other parameters in the model.

\subsection{Linear Regression using Gaussian Basis Function}

Polynomial linear regression model given by (1) is a linear function of input variables $\left(z_{i}\right)$ and thus forces significant limitation on the model. Therefore, the model presented in the previous section can be further investigated by operating with fixed non-linear function given by (7) 
International Journal of Mathematical, Engineering and Management Sciences

Vol. 5, No. 6, 1284-1299, 2020

https://doi.org/10.33889/IJMEMS.2020.5.6.095

$V_{P}(z, \alpha)=\sum_{j=0}^{M-1} \alpha_{j} \phi_{j}(z)$

where $\phi_{j}(z)$ is the basis function, index $j=0,1 \ldots . . M-1, M$ is the total number of parameters, $\alpha=\left(\alpha_{0} \ldots \ldots . \alpha_{M-1}\right)^{T}$ and $\phi=\left(\phi_{0} \ldots \ldots \ldots \phi_{M-1}\right)^{T}$. In this paper, we have considered Gaussian basis function as given in (8)

$\phi_{j}(z)=\exp \left\{-\frac{\left(z-\mu_{j}\right)^{2}}{2 s^{2}}\right\}$

$\mu_{j}$ controls the locations of the basis functions in the input space, and the parameter $s$ is the width or spatial space.

The experimental dataset is fitted by minimizing the sum of the squares error function. A dataset of axial position is considered as input $Z=\left\{z_{1}, z_{2} \ldots \ldots . . z_{n}\right\}$ with the corresponding target plasma potential values. Target values are grouped in the column vector. The $z$ values appear as conditioning variables. The data points are drawn independently and shown in the Figure 1. Sum of the squared error function is defined by (9)

$E_{D}(\alpha)=\frac{1}{2} \sum_{n=1}^{N}\left\{z_{n}-\alpha^{T} \phi\left(z_{n}\right)\right\}^{2}$

A regularization term $E_{w}(\alpha)=\frac{1}{2} \alpha^{T} \alpha$ is introduced to control over-fitting, so the total error function to be minimized is computed as (10)

$E(\alpha)=\frac{1}{2} \sum_{n=1}^{N}\left\{z_{n}-\alpha^{T} \phi\left(z_{n}\right)\right\}^{2}+\frac{\lambda}{2} \alpha^{T} \alpha$

$E_{w}(\alpha)$ is also known as weight decay. It has the advantage that the error function remains a quadratic function of $\alpha$. A tuning parameter $(\lambda)$ controls the strength of the penalty term. When $\lambda=$ 0 , ridge regression equals least squares regression. If $\lambda=\infty$, all coefficients are shrunk to zero. The ideal penalty is, therefore, somewhere in between 0 and $\infty$. It shrinks the parameters; therefore, it is mostly used to prevent multicollinearity, and it also reduces the model complexity by coefficient shrinkage.

\section{Result and Discussion}

\subsection{Measurement of Plasma Parameters}

In the present study, hydrogen helicon plasma is generated using the Nagoya type-III antenna. The helicon hydrogen gas plasma is obtained by operating Nagoya type-III antenna at RF power $\geq$ 400W. A sudden jump after 400W can be seen in Figure 2 which shows that a stable helicon plasma 
International Journal of Mathematical, Engineering and Management Sciences

Vol. 5, No. 6, 1284-1299, 2020

https://doi.org/10.33889/IJMEMS.2020.5.6.095

mode exists after $700 \mathrm{~W}$. For the presented study, the experimental dataset obtained at 6 mTorr pressure and B field 86 Gauss is considered for developing a regression model for axial plasma potential variations.

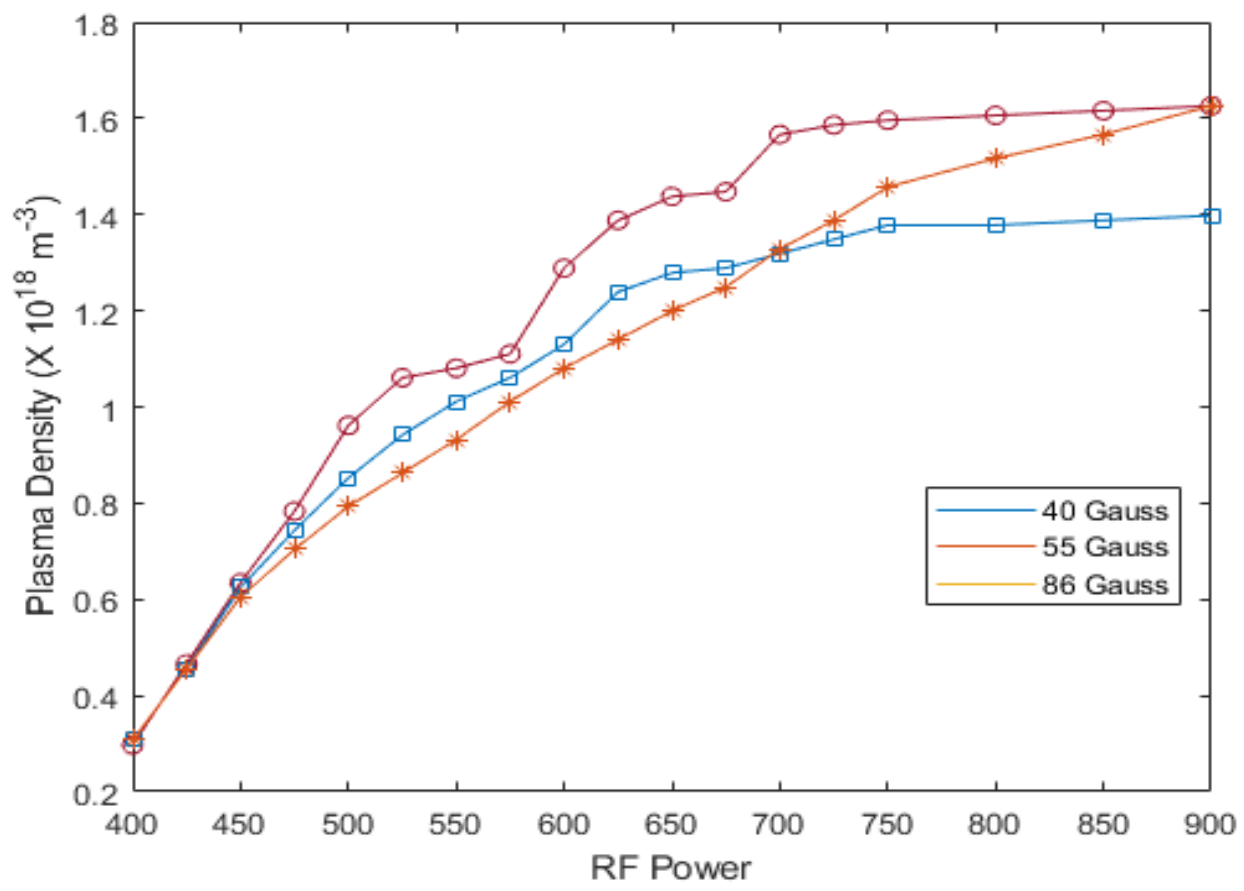

Figure 2. Variation of ion saturation current with increasing RF power (Watts) at 6-mTorr pressure

\subsection{Polynomial Regression Model}

Several orders of the polynomial by keeping the axial position as the independent variable are trained with the training data set. Considering the non-linearity and randomness in the experimental data shown in Figure 1, we started regression analysis by initiating with $5^{\text {th }}$ order polynomial. The $1-\mathrm{D}$ independent variable is transformed into a 2-D vector of the $5^{\text {th }}-8^{\text {th }}$ order. Figure 3 shows the results of the polynomial regression. It can be observed that lower-order polynomials show the poor curve fitting, e.g. $5^{\text {th }}$ order polynomial curve fitting shows an abrupt jump towards the end of the plot which may give arbitrary value when operated with new data set. Moreover, the nature of curve fitting improves when the order of the polynomial is further increased. The experimental data set is used for training and testing the prediction results. The accuracy of results is measured by root mean squared error and the $\mathrm{R}^{2}$ value or coefficient of determination. The $\mathrm{R}$ squared value measures the model performance relative to a simple mean of the target values. 
International Journal of Mathematical, Engineering and Management Sciences

Vol. 5, No. 6, 1284-1299, 2020

https://doi.org/10.33889/IJMEMS.2020.5.6.095

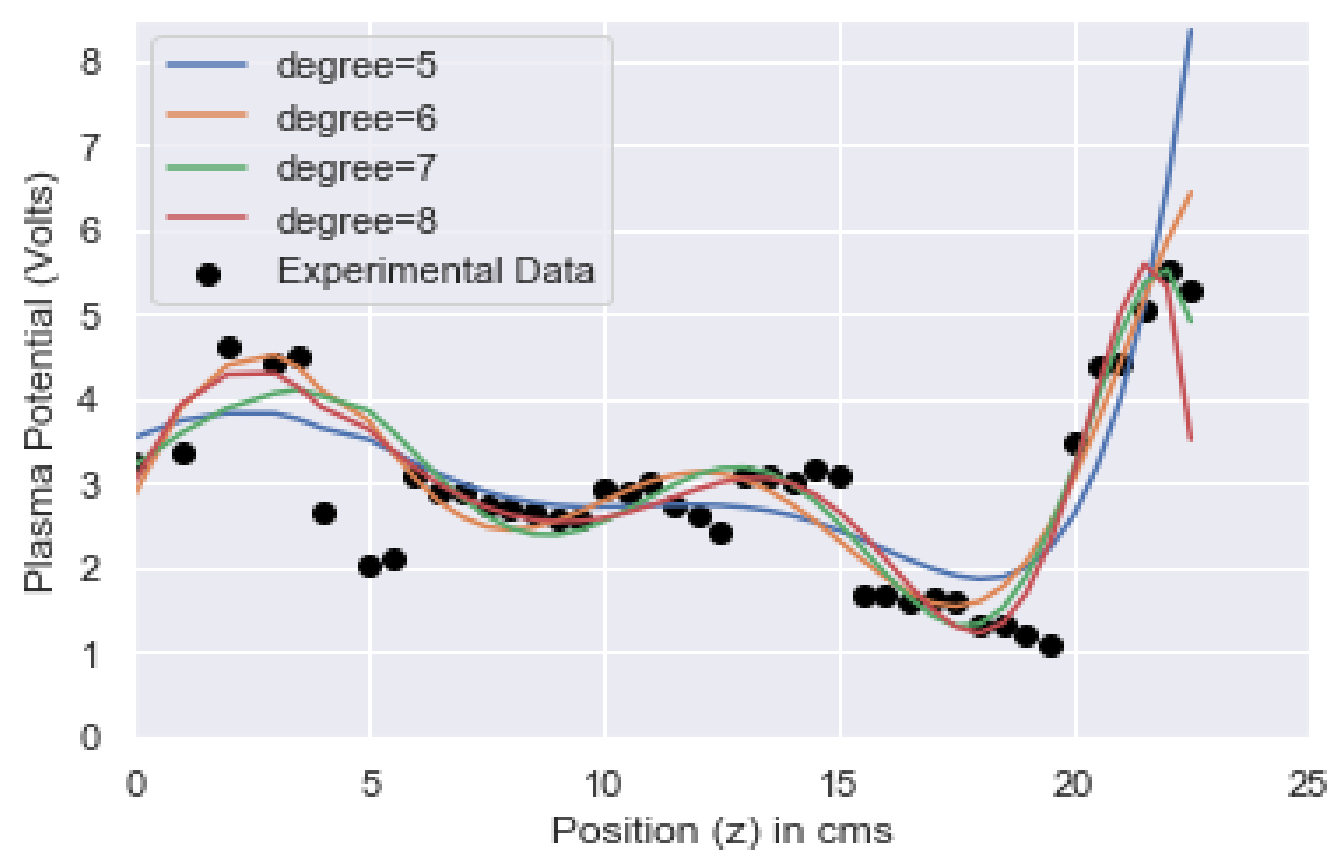

Figure 3. Prediction of axial variation of plasma potential using the polynomial regression model

$V_{p}=+2.994+0.0001+2.383 z-1.475 z^{2}+0.398 z^{3}-0.062 z^{4}+0.006 z^{5}-0.000 z^{6}+0.000 z^{7}-0.000 z^{8}(11)$

The careful observation of results shown in Table 2 suggests that $8^{\text {th }}$ order polynomial given in (11) fairly presents the axial plasma potential variation for HELEN-I.

Table 2. Model performance comparison for the polynomials of degree $5^{\text {th }}$ to $8^{\text {th }}$

\begin{tabular}{|c|c|c|c|c|}
\hline \multirow{2}{*}{$\begin{array}{c}\text { Degree of } \\
\text { Polynomial }\end{array}$} & \multicolumn{2}{|c|}{ Model Performance for Training Set } & \multicolumn{2}{c|}{ Model Performance for Test Set } \\
\cline { 2 - 5 } & RMSE & $\mathrm{R}^{2}$ & RMSE & $\mathrm{R}^{2}$ \\
\hline 5 & 0.701 & 0.573 & 0.787 & 0.545 \\
\hline 6 & 0.544 & 0.743 & 0.950 & 0.338 \\
\hline 7 & 0.488 & 0.793 & 0.756 & 0.581 \\
\hline 8 & 0.454 & 0.820 & 0.659 & 0.682 \\
\hline
\end{tabular}

We may notice that the coefficient values for $z^{6}, z^{7}$ and $z^{8}$ are converging to small values $(\approx 0)$ suggests the saturation of learning for the model. Model performance shows the significant difference between the RMSE values for the training set and test set. Similar inconsistency in the results can also be observed through the training and validation curve shown in Figure 4. 


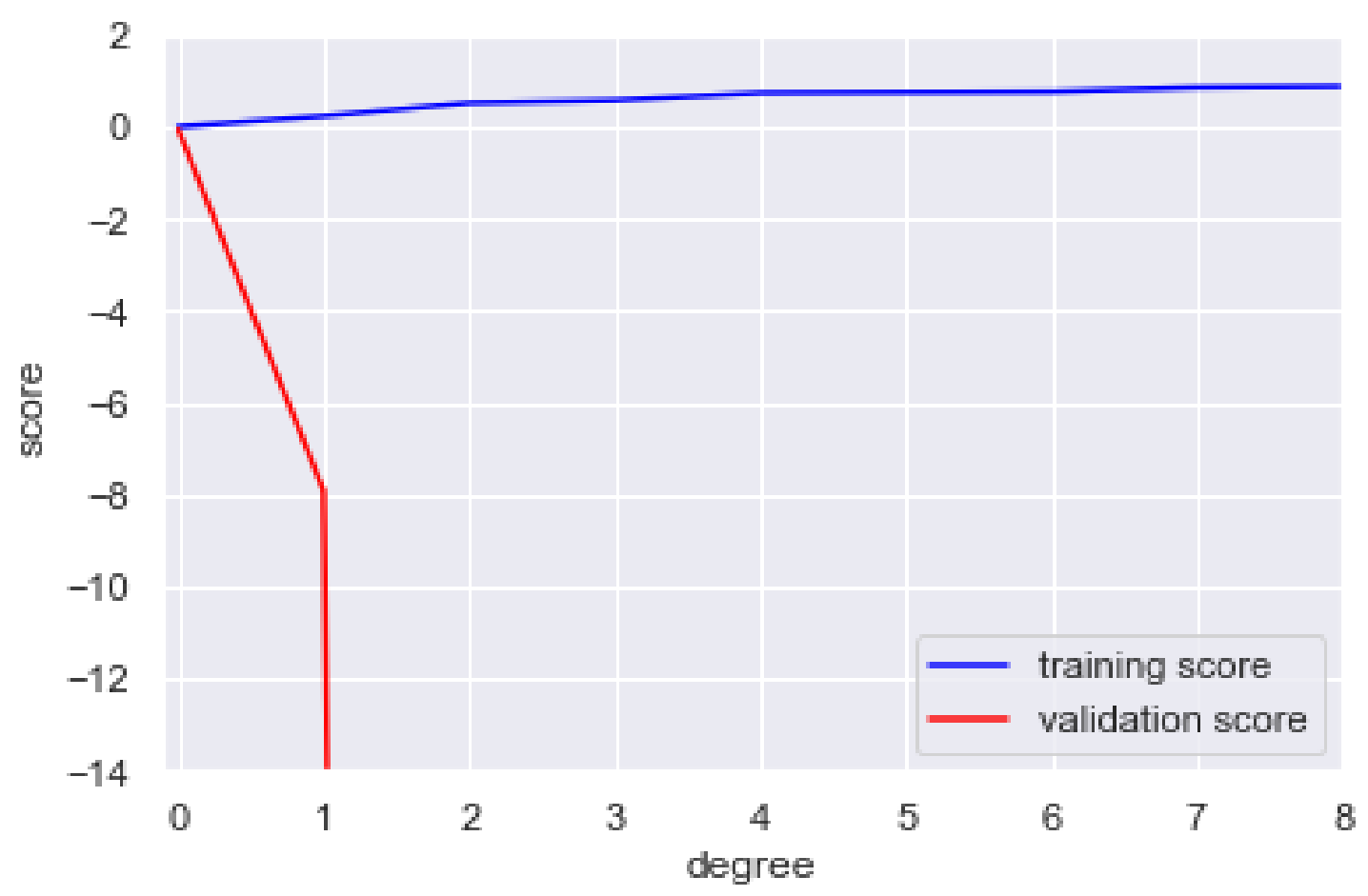

Figure 4 . The variance scores for the polynomials of the degree $\left(5^{\text {th }}-8^{\text {th }}\right)$

The diverging nature of the validation curve shows high variance value between the training and test data set. This necessitates the implementation of Gaussian basis function-based linear regression. The detailed discussion on the results obtained by implementing GBF linear regression is given in the next section.

\subsection{Gaussian Basis Function Model}

Based on the literature reviewed, scaled Gaussian basis functions can be the promising candidate for prediction of axial variation of plasma potentials. Multiple numbers of Gaussian basis functions are summed up, which efficiently fit the nature of the experimental dataset. For the available experimental data set of axial position and plasma potentials, 15, 20, 30 and 40-dimensional Gaussian basis functions or base estimators are introduced into the linear model given in (8). Figure 5 shows the prediction results of the GBF regression model.

It is clear from the Figure 5 that the introduction of basis functions into the linear model given in (7) makes the model much more flexible and accurate, but it also can very quickly lead to overfitting. Careful investigation of Figure 5 towards the connection between 2 data points seems to be linear, which is contrary to the inherent nature of GBF. The reason for linearity present between two data points can be visualised by plotting the coefficients $(\alpha)$ given in (9) and the basis function. Figure 6 shows the amplitude for 15, 20, 30 and 40-dimensional basis functions concerning the respective prediction plots. Here, coefficients $(\alpha)$ are attaining substantial values which simply mean typical over-fitting behaviour, and it occurs when basis function overlap: the coefficients of adjacent basis functions cancel each other. In other words, some predicted values are exceeding the 
actual values which need to be brought down. The problem arose due to the cancellation of adjacent coefficients is removed by keeping tuning parameter $(\lambda=2)$ to penalise large values of the model parameters known as regularisation given in (10) as $E_{w}(\alpha)$.

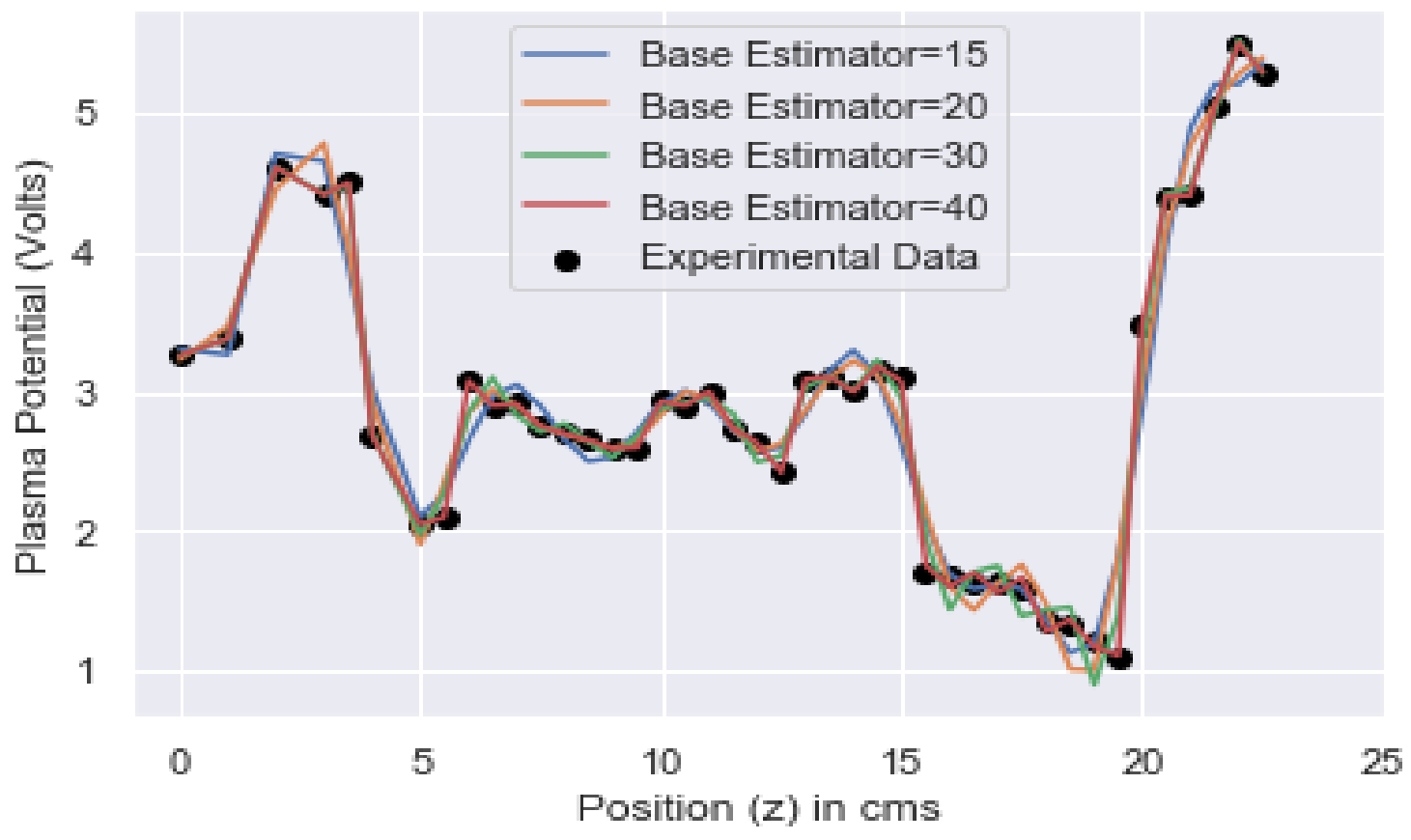

Figure 5. Prediction of axial plasma potential variation using GBF regression model

Figure 7 clearly shows the reduction in coefficient values with increasing dimensionality. The coefficient values typically lie between -1 to 1 . The comparison of Figure 6 and 7 show that the coefficients $(\alpha)$ are significantly reduced from the range $\pm 10^{11}$ to \pm 1 . Due to the hefty penalty applied the sharp edges presented in the predicted values of $V_{p}$ shown in Figure 6 are reduced hence resulted in overall improvised predicted $V_{P}$ values as shown in Figure 7.

After adding the penalty to the sum of the squared error function given in (9), the prediction results for the same range of Gaussian basis dimension values show the smoothening of between two consecutive data points as shown in Figure 8. The regularized GBF regressor model produces an excellent fit to the experimental data of the axial plasma potential variation shown in Figure 8.

The model performance is estimated through the RMSE and $\mathrm{R}^{2}$ values. The model performance in terms of RMSE and $\mathrm{R}^{2}$ for 15, 20, 30, and 40 dimension GBF regressor is given in Table 3. 

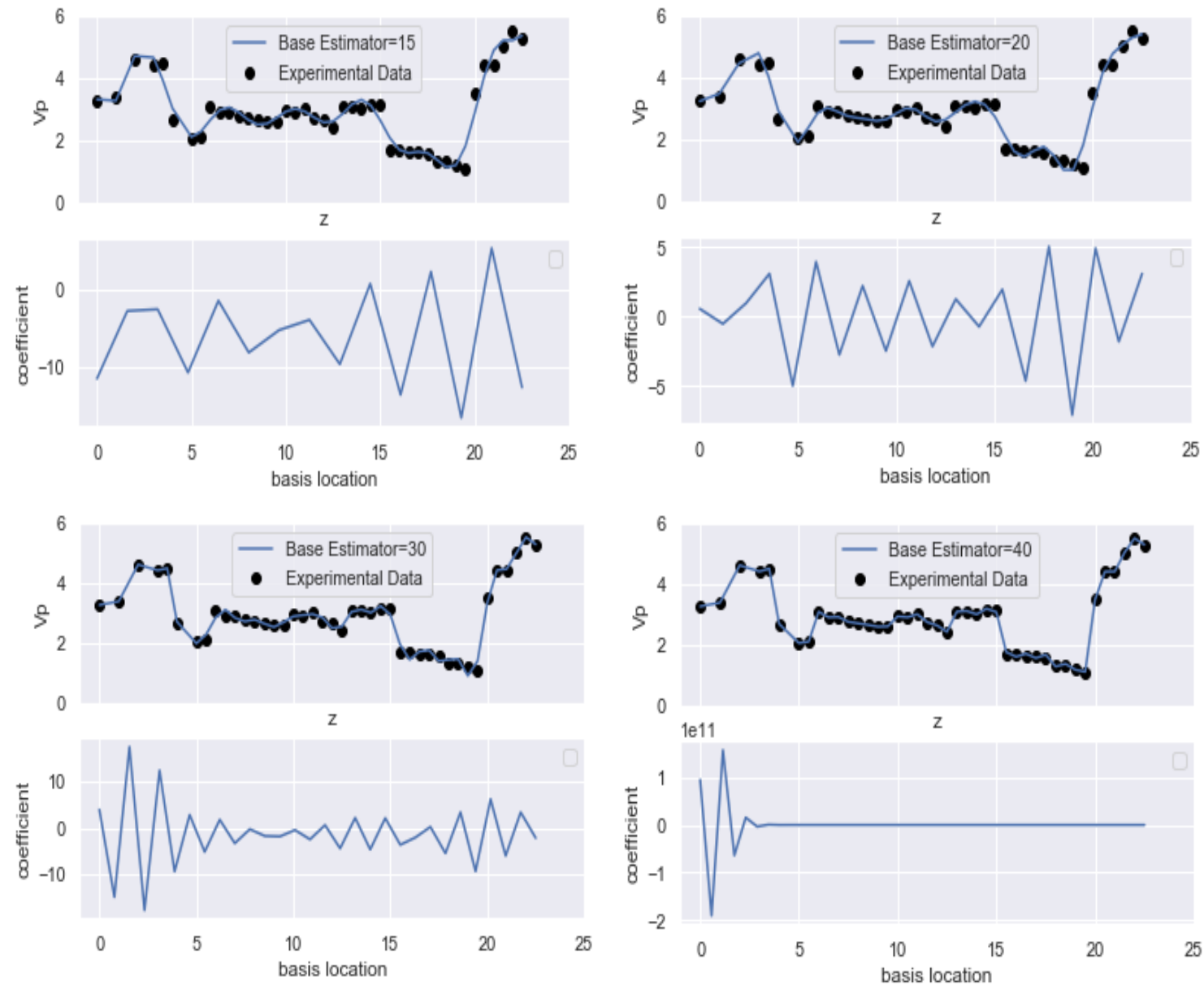

Figure 6. The coefficients of adjacent Gaussian basis functions (of Base Estimator $=15,20,30,40$ ) for the prediction plots

RMSE and $R^{2}$ value for the training set for the polynomial of degree $\left(5^{\text {th }}-8^{\text {th }}\right)$ is less than the RMSE value for the test set. However, with an increasing degree of the polynomial trade-off between the bias and variance increases (e.g. degree 6). On the other hand, results shown in Figure 9 shows a bright improvement in the performance parameters, where RMSE score is gradually decreasing, and $\mathrm{R}^{2}$ scores are increasing form the training and the test set, respectively. Moreover, this pattern is not observed for the polynomial regression model.

Hence, the GBF regressor model (40-Dimensional) can fairly represent the axial plasma potential variation and can be used for predicting the plasma potential without conducting real-time experiments on the experimental setup.

Figure 9 shows the graphical model of performance measured through performance parameters (RMSE and $\mathrm{R}^{2}$ values) for the polynomial regression model and regularized GBF regression model. 
International Journal of Mathematical, Engineering and Management Sciences

Vol. 5, No. 6, 1284-1299, 2020

https://doi.org/10.33889/IJMEMS.2020.5.6.095
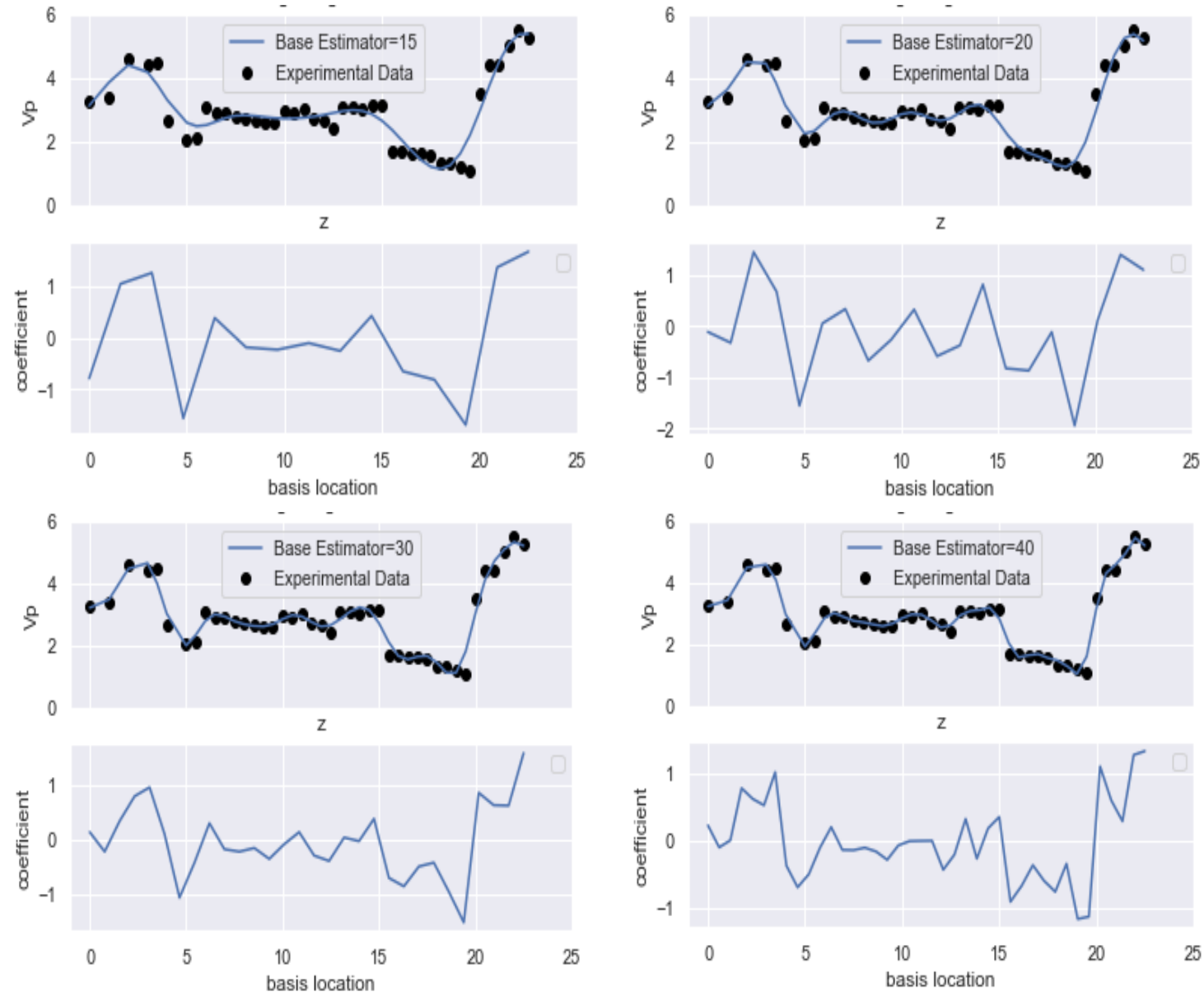

Figure 7. The regularised coefficients of adjacent Gaussian basis functions (Base Estimator $=15,20,30,40$ ) for the prediction plots

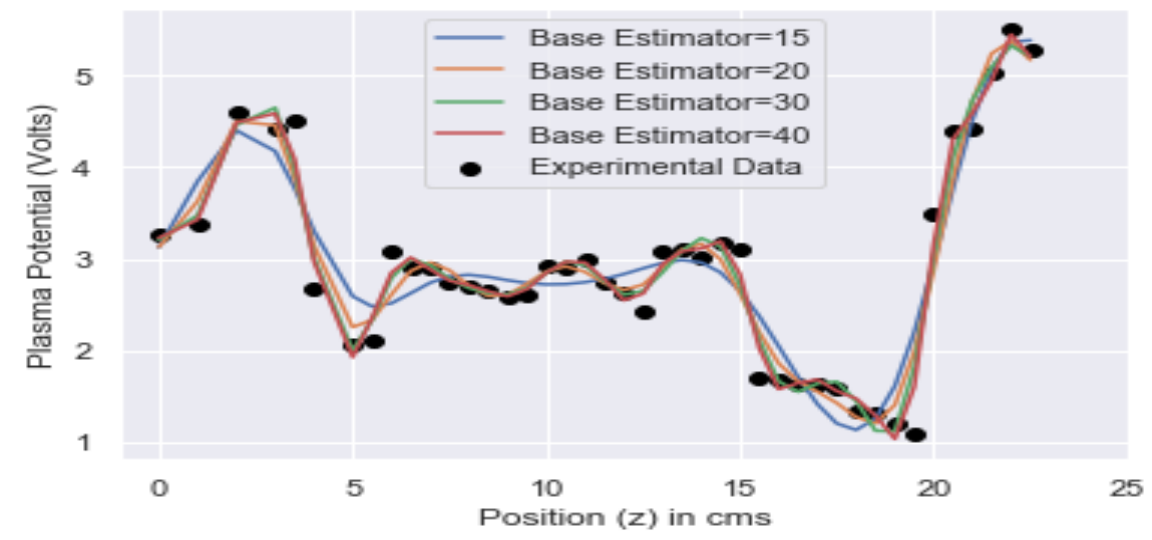

Figure 8. Prediction of axial plasma potential variation using regularised GBF regression model 
International Journal of Mathematical, Engineering and Management Sciences

Vol. 5, No. 6, 1284-1299, 2020

https://doi.org/10.33889/IJMEMS.2020.5.6.095

Table 3. Model performance comparison for regularised Gaussian Basis Regression Model

\begin{tabular}{|c|c|c|c|c|}
\hline \multirow{2}{*}{ Base Estimators } & \multicolumn{2}{|c|}{ Model Performance for Training Set } & \multicolumn{2}{c|}{ Model Performance for Test Set } \\
\cline { 2 - 4 } & RMSE & $\mathrm{R}^{2}$ & RMSE & $\mathrm{R}^{2}$ \\
\hline 15 & 0.2589 & 0.9451 & 0.3600 & 0.9013 \\
\hline 20 & 0.2313 & 0.9562 & 0.2988 & 0.9320 \\
\hline 30 & 0.1250 & 0.9872 & 0.2222 & 0.9624 \\
\hline 40 & 0.0292 & 0.9993 & 0.1645 & 0.9793 \\
\hline
\end{tabular}
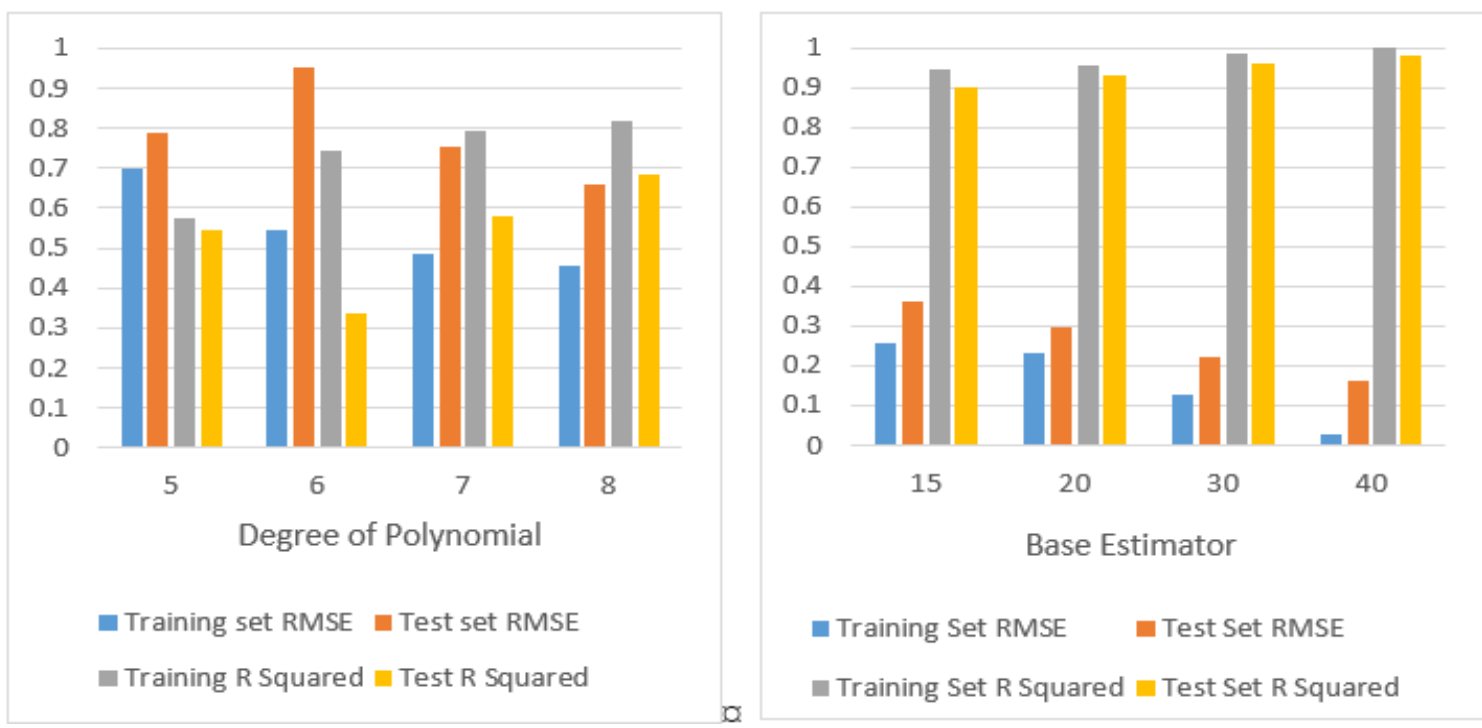

Figure 9. The performance of polynomial linear regression model for 5,6,7, and 8 degrees of polynomials $(5,6,7$, and 8) and GBF regression model performance for 15, 20, 30, and 40 dimension GBF regressor

\section{Conclusion and Future Work}

The present paper explores the application of linear regression-based machine learning technique for predicting axial variation of plasma potential. Further, this paper establishes that the machine learning-based regression techniques may be proved as a promising tool in diagnostics for plasma device/ion sources.

- The paper successfully employs polynomial linear regression and linear regression using Gaussian basis function for predicting the axial variation of plasma potential.

- Based on the analysis carried out in this study, the Gaussian basis regressor model turns out to be a better candidate for predicting the plasma potential as compared to the polynomial regressor model.

- The methodology presented in the paper can be further utilized for predicting other plasma parameters such as ion saturation current, plasma temperature and plasma density in future. 
International Journal of Mathematical, Engineering and Management Sciences

Vol. 5, No. 6, 1284-1299, 2020

https://doi.org/10.33889/IJMEMS.2020.5.6.095

\section{Conflict of Interest}

All the authors have equal contribution to this work, and it is declared that there is no conflict of interest for this publication.

\section{Acknowledgements}

The Authors sincere acknowledge the Institute for Plasma Research, Department of Atomic Energy, Government of India for allowing the authors to utilize their facility for conducting experiments and generating data set for the present study.

\section{References}

Bhattacharyya, R. (2009). Indigenous ion sources for material processing. Defence Science Journal, 59(4), 377-394.

Bishop, C.M. (2006). Pattern recognition and machine learning. Springer Science+Business Media. New York.

Gidon, D., Pei, X., Bonzanini, A.D., Graves, D.B., \& Mesbah, A. (2019). Machine learning for real-time diagnostics of cold atmospheric plasma sources. IEEE Transactions on Radiation and Plasma Medical Sciences, 3(5), 597-605.

Godyak, V.A., \& Alexandrovich, B.M. (2015). Comparative analyses of plasma probe diagnostics techniques. Journal of Applied Physics, 118(23), 233302.

Ho, A., Citrin, J., Auriemma, F., Bourdelle, C., Casson, F.J., Kim, H.T., Manas, P., Szepesi, G., \& Weisen, H. (2019). Application of Gaussian process regression to plasma turbulent transport model validation via integrated modelling. Nuclear Fusion, 59(5), 056007.

Hopkins, M.B. (1995). Langmuir probe measurements in the gaseous electronics conference rf reference. Journal of Research of the National Institute of Standards and Technology, 100(4), 415-425.

Kates-Harbeck, J., Svyatkovskiy, A., \& Tang, W. (2019). Predicting disruptive instabilities in controlled fusion plasmas through deep learning. Nature, 568(7753), 526-531.

Kim, B., Lee, D.W., Park, K.Y., Choi, S.R., \& Choi, S. (2004). Prediction of plasma etching using a randomised generalised regression neural network. Vacuum, 76(1), 37-43.

Kondor, R. (2004). Regression by linear combination of basis functions. Thesis, School of Computer Science, University of St. Andrews. New York

Lichtenberg, J.M. \& Şimşek, Ö. (2017). Simple regression models. Proceedings of the NIPS 2016 Workshop on Imperfect Decision Makers in PMLR, 58, 13-25.

Merlino, R.L. (2007). Understanding Langmuir probe current-voltage characteristics. American Journal of Physics, 75(12), 1078-1085.

Mesbah, A., \& Graves, D.B. (2019). Machine learning for modeling, diagnostics, and control of nonequilibrium plasmas. Journal of Physics D: Applied Physics, 52(30), 30LT02.

Ostertagová, E. (2012). Modelling using polynomial regression. Procedia Engineering, 48, 500-506.

Palmer, P.B., \& Connell, D.G.O. (2009). Regression analysis for prediction: understanding the process. Cardiopulmonary Physical Therapy Journal, 20(3), 23-26.

Pandey, A., Mukherjee, D., Borah, D., Bandyopadhyay, M., Tyagi, H., Yadav, R., \& Chakraborty, A. (2019). Characterisation of hydrogen plasma in a permanent ring magnet based helicon plasma source for negative ion source research. Plasma Physics and Controlled Fusion, 61(6), 065003. 
International Journal of Mathematical, Engineering and Management Sciences

Vol. 5, No. 6, 1284-1299, 2020

https://doi.org/10.33889/IJMEMS.2020.5.6.095

Pandey, A., Bandyopadhyay, M., Sudhir, D., \& Chakraborty, A. (2017). Performance evaluation of a permanent ring magnet based helicon plasma source for negative ion source research. Review of Scientific Instruments, 88(10), 103509.

Ramesha, K., \& Raja, K.B. (2011). Gram-Schmidt orthogonalization based face recognition using DWT. International Journal of Engineering Science and Technology, 3(1), 494-503.

Rea, C., Granetz, R.S., Montes, K., Tinguely, R.A., Eidietis, N., Hanson, J.M., \& Sammuli, B. (2018). Disruption prediction investigations using machine learning tools on DIII-D and Alcator C-Mod. Plasma Physics and Controlled Fusion, 60(8), 084004.

Schneider, A., Hommel, G., \& Blettner, M. (2010). Linear regression analysis: part 14 of a series on evaluation of scientific publications. Deutsches Arzteblatt International, 107(44), 776-782.

Witman, M., Gidon, D., Graves, D.B., Smit, B., \& Mesbah, A. (2019). Sim-to-real transfer reinforcement learning for control of thermal effects of an atmospheric pressure plasma jet. Plasma Sources Science and Technology, 28(9), 95019. 